\title{
Substitutional Reality: a Research Agenda
}

\author{
Adalberto L. Simeone* \\ University of Portsmouth
}

\begin{abstract}
In our previous work on Substitutional Reality in the context of Virtual Reality we presented an exploration of a class of Virtual Environments where every physical object surrounding the user is associated with appropriate virtual counterparts. Differently from "passive haptics", Substitutional Reality assumes the existence of a discrepancy in the association. This previous work explored how far this mismatch can be pushed and its impact on the believability of the experience.

In this paper we discuss three main research directions for Substitutional Reality. Firstly, the design space is largely unexplored as the initial investigation focused on the mismatch between real and virtual objects. Secondly, the development of systems enabling a dynamic substitution process represents a key challenge. Thirdly, we discuss how to approach the design and evaluation of these experiences.
\end{abstract}

Index Terms: H.5.1 [Information Interfaces and Presentation]: Artificial, augmented and virtual realities-

\section{INTRODUCTION}

Substitutional Reality (SR) [12] builds on the concept of associating virtual objects to physical proxies surrounding the user. The resulting substituted Virtual Environment or Substitutional Environment (SE), would be based on the physical environment in which the user is, but would look radically different. What differentiates SR from previous work on passive haptics [5] in the Virtual Reality literature, is the existence of a mismatch in this pairing. In our previous work, we have studied the extents of this mismatch in two user studies. From the results, we derived a set of guidelines supporting future designers of SR systems. In this paper, we discuss new future research directions.

Further research on Substitutional Reality needs to tackle three different challenges: firstly, investigate in greater detail the SR design space; secondly, develop prototype SR systems capable of generating an SE based on the physical environment in which it is deployed; thirdly, explore how to best convey and evaluate abstract representations of a Virtual Environment that can then be adapted by an SR system to the requirements of each physical environment.

Indeed, our first work represents an initial exploration of the SR design space. We defined a layered model of potential substitutions which allowed us to explore the factors affecting the believability of the experience. From a conceptual point of view, there are several aspects that would be complementary to our first exploration. Our experiment focused on the mismatch between physical objects and their virtual counterparts. As such, it did not involve the possibility of really walking in the Substituted Environment. Indeed, one of the greatest challenges of VR comes from the disparity between the simulated environment and the physical space in which the experience takes place. If there is no correspondence, walking in reality would pose safety issues due to the risk of bumping into walls or furniture users might not be aware of. While immersed in SE, users

*e-mail: adalberto.simeone@port.ac.uk would be aware that the virtual objects they see are matched to tangible objects. The awareness of these virtual objects delimiting the space in which they can move represents a way to untether users from the limitations of desktop-style VR. We also discuss other aspects that warrant further investigation in SR: such as exploring in greater detail the dimensions introduced by the model, how to combine immaterial and substituted objects, and how to approach multiuser SEs where users themselves are also subjected to a process of substitution.

The second issue focuses on the technical challenges of SR. In our previous work, the environments in which the users were immersed were manually authored. However, a real-world application of Substitutional Reality, would require a system capable of dynamically producing a Substitutional Environment based on the physical environment given as input. As there are no existing systems capable of performing this process, it is unclear what kind of issues might arise and their implications on interaction and locomotion. We believe that research on SR systems could progress in incremental steps by first focusing on attainable goals such as dynamic substitution of the user's surroundings (i.e. desktop SR), and then proceeding to tackle larger environments such as rooms and beyond. Once prototype SR systems are introduced, research can progress on studying advanced issues such as the combination of SR with mid-air haptics rendering systems.

The last issue concerns the design and evaluation of SR experiences. Content creators will have to design SR experiences without knowing precisely in which physical environment they will be experienced. They could be vastly different in terms of both layout and object availability. We discuss how to approach this problem and the issue of evaluating different instances of the same SR experience.

\section{Related Work}

The term Substitutional Reality was originally introduced by Suzuki et al. [15]. However, they focused on studying the impact of the alternation of live and recorded scenes on unaware users who were wearing a Head-Mounted Display. In the context of Virtual Reality, Substitutional Reality refers to a class of Virtual Environments where every physical object surrounding the user has been paired to a virtual object, on the assumption that a mismatch exists between the two [12].

Other works have focused on augmenting users' own domestic environments to provide more compelling experiences. For example, Illumiroom is a proof-of-concept system by Jones et al. [8]. The system envisions the use of projected imagery to augment gaming experiences played on a regular display. It calibrates itself on the layout of the room in which it is installed and projects peripheral imagery. In this way, it can change the appearance of a room or extend the field of view of a game. For example, the area around the display can be used to show the rest of the game's environment or extend a grenade's bounce outside the display and into the room.

RoomAlive [7] is another proof-of-concept system that uses projected imagery to create a gaming experience in the user's own living room. By reconstructing the main layout of the room in which the system is deployed, the system is able to identify planar surfaces. It can then project virtual objects and characters that users can interact with in game scenarios. For example, in a whack-amole scenario, virtual "moles" are rendered on the room's walls or 

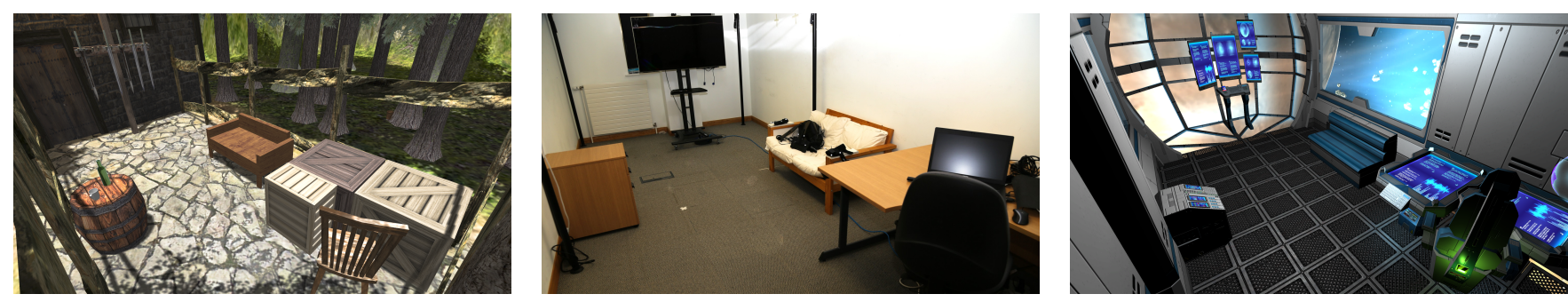

Figure 1: Two Substitutional Environments from our previous work [12] based on the same physical living room: a medieval courtyard (left) and the bridge of a spaceship (right).

floors. The user can interact with them by whacking and shooting.

GravitySpace [1] is a system that, by means of a pressuresensitive floor (which also acts as a display), is able to recreate a Virtual Environment based on what is happening above the floor. Using image-recognition techniques, the system analyses the contact points of furniture and people. Furniture are detected through their pressure and are uniquely identified by a rotation-invariant marker. Users are detected by matching their shoe footprint to a database of registered shoes. In this way the system is able to support various applications such as interactive games.

\section{Perception in Substitutional Reality}

The model described in our previous work [12] identified a set of potential substitution types. This previous work explored the impact of each type, resulting in the identification of high-level factors that affect the user experience. From this starting point, there are three possible research directions in which further research is needed:

Model - In order to gain a deeper understanding of the design space of SR, it would be necessary to pursue further research in this direction. Is the model exhaustive? Are there other types of substitutions? Furthermore, it is necessary to investigate each layer of the model in greater detail. For example, in aesthetic substitutions, which type of materials have the least impact on the suspension of disbelief? At which point does a mismatch in size become significant? Considering the mismatch between the affordances [4] portrayed by the virtual object and those related to its physical proxy, are there cases of mismatch that have more impact than others?

Locomotion - Physically walking in a Virtual Environment is one of the overarching goal of Substitutional Reality and Virtual Reality [?]. As previously described, substitution will also incur with larger objects and architectural elements. It will thus be necessary to study the impact of the different types of substitution described in the model when applied to an environment in which users can physically move. There are a number of potential issues, for example: how to allow users to move in a Virtual Environment much larger than the space allowed by the physical environment. Past work have studied Redirected Walking techniques as a solution to this problem. Variations of these techniques could be applied to SR as well, however this could lead to participants experiencing the same obstacle patterns across different Virtual Environments, and it is unclear if this will negatively affect the sense of presence.

Combining immaterial and substituted objects - In our previous work [12], we found that objects that users are least likely to interact with can be subjected to substitutions having a greater mismatch. Indeed, objects that are out of reach could not even be matched to any physical element at all. However, there might be some situations in which an SR experience requires the portrayal of an element that cannot be matched to a physical object, which the user might interact with. For example, a protective force field surrounding an object the user needs to obtain. How can the suspension of disbelief be maintained in these situations? If the aforemen- tioned force field looks convincingly dangerous, from a graphical perspective, would it suffice to discourage users to interact with it and thus avoid breaking the illusion?

Substitution of People - Current technology allows systems to detect the posture of an user relatively easy. Given this information, it becomes then possible to assign a virtual avatar to substitute a real person in a co-operative scenario. Past work on Collaborative Virtual Environments (CVEs) has mostly focused on distributed networked telepresence, whereas co-located multi-user Virtual Reality systems have received less attention [10]. Game-like scenarios would be a suitable context in which to explore the combination of Substitutional Reality and multi-user Virtual Reality. If we imagine a group of 2 or more friends playing an SR game or visiting an SR version of a museum exhibit, there are various challenges to overcome:

- Acceptance - In the two studies performed in [12] participants were asked whether they would prefer to participate in SR experiences alone or with friends; results were mixed, therefore it would be necessary to investigate which factors can affect user's view of multi-user SR experiences.

- Safety - Can SR experiences safely involve scenarios in which players compete against each other (i.e. a swordfighting game)? While this issue might not be exclusive to multi-user scenarios (i.e. accidentally hitting objects or interacting with those who appear less fragile than they are in reality), it raises the question of how best to approach it in the context of SR.

- The role of non-participants - It is conceivable that people (or even pets) who are not participating in an SR experience might enter the physical environment in which the SR system is deployed. As such they might interact with the environment. Seeing an object in the Virtual Environment moving on its own might negatively affect the sense of presence. Analogously, if they are not represented in the environment, they effectively become obstacles that need to be avoided.

User Interfaces - The role of user interfaces is a challenging issue that is also relevant to Virtual Reality systems. However, in addition to the challenges of designing 3DUIs for system-control tasks, there are also some specific issues that would arise in SR experiences. For example, how can users change the status of the environment? Considering the scenario of a chest substituting a physical box, there exists a functional mismatch between the two. In the VE, the chest might be portrayed to be open in a different fashion than the box, or it might not be possible to open it at all. This mismatch can be mitigated by designing UIs that fill these gaps. For example, the previously described problem can be solved by presenting users with an inventory screen depicting the (entirely virtual) contents of said chest. This however leads back to the problem of combining objects that do not have an associated physical proxy and those who do, in the same Substitutional Environment. 


\section{Technical Challenges}

The development of real-time Substitutional Reality systems is one of the main research directions to pursue. There are two main challenges: 1) the development of a system capable of performing the Substitution process, i.e. dynamically adapting a Virtual Environment based on a physical environment given as input; 2) improving the tracking of users and objects in domestic environments.

\subsection{Substitutional Reality Systems}

We envision several types of SR systems, whose complexity increases with the scale of the physical environment they attempt to substitute. These are:

- Desktop SR - In some scenarios it may be sufficient to simulate only the user's immediate surroundings. For example, in a racing or flight simulator the user is not expected to leave the car interior or cockpit during the duration of the simulation. Such an SR system would need to focus only on matching the objects available on the desktop and those needed by the Virtual Environment. Users would not be able to walk within the simulated environment.

- Room-Sized SR - Substitution of room-sized domestic environments would be the logical next step. An SR experience would take place in a room chosen by the user. The SR system would adapt the Virtual Environment required by the experience to the space available in the physical environment. The system would need to track non-static objects that can be moved.

- Large-Scale SR - If we assume that Room-Sized SR needs the support of static tracking devices, substituting any kind of environment in which a SR system is deployed could represent the ultimate goal. This would require new research on sensing devices in order for SR to remain non-intrusive.

In the short to medium term, we believe Desktop and Room-Sized SR to be the most feasible and achievable goals, with Large-Scale SR driving the overall vision. Kinect Fusion [6] is a real time reconstruction system that, through a depth-camera, is able to reconstruct the point-cloud of the environment it is used in. In some applications, it is necessary to reconstruct an environment with as much fidelity as possible, for example for Telepresence or Augmented Reality [3]. However, in the context of Substitutional Reality, a coarser representation might suffice. The goal of this substitution process would not to be the building of a Virtual Environment acting as a replica of the real environment the user is in, but the creation of one that uses its physical elements to provide tangibility to a radically different Virtual Environment. We envision a substitution process consisting of several steps:

1. Retrieve the Point Cloud of the physical environment to substitute.

2. Detect the main features of the environment (e.g., the floor, walls, and ceiling, see Fig. 5).

3. Segment furniture and other objects.

4. Fit bounding boxes on each segmented object (see Fig. 5).

5. Detect semantic information.

6. Match physical objects with virtual objects required from the Substitutional Reality experience.
Our initial experimentations are based on the analysis of a static point cloud frame, using the Point Cloud Library [11]. After removing the planes constituting the floor, ceiling and walls, the remaining point cloud represents the objects in the room. A variety of segmentation algorithms can be used to divide them into clusters (see Fig. 5). Once objects are separated into clusters (on a coarse scale), it would be possible to fit bounding boxes onto each.

Beyond the implementation of an SR system, a further goal would be the artificial simulation of the mismatch between physical and virtual objects. For example, projects like Aireal [14] and Ultrahaptics [2] allow users to sense mid-air haptic feedback. Further research in that direction could investigate how to use a physical proxy and render its missing features, in order to enhance the believability of the illusion. For example, given a real torch as proxy for a Lightsaber, it is conceivable that the real object would lack some features of the virtual object, i.e. an example of "addition/subtraction" of features. Ideally, the haptic system would render the missing features of the Lightsaber, such as the activation switch, ridges in the hilt, etc.

\subsection{User and Object tracking}

An SR system will require technologies capable of sensing the user's position by using devices that do not require a radical augmentation of the physical environment. A number of consumer fullbody motion tracking devices are being developed with the intent of reducing the cost of this technology, for example STEM [13] and PrioVR [16].

Tracking objects users wish to interact with represents the other side of this challenge. Real-time object segmentation and classification might be the long-term goal. However, in the shorter term individually tracking objects users explicitly wish to be interactive could represent a more cost-effective solution. Indeed, this method has the advantage of not needing to infer semantic information from its visual representation, as the user would label it manually. However, the disadvantage consists in the physical tangibility of the sensor itself which could negatively affect the sense of presence. In our previous work, we placed IR markers on the parts of the objects that were less likely to be interacted with such as a mug's top or a bottle's neck.

\section{Design and Evaluation of SR Experiences}

Once proof-of-concept SR systems are available, designing SR experiences would be the next challenge to explore. An SR experience, be it game-oriented or educational in nature, should describe tasks or activities the participants can perform while immersed in the system. However, in conventional games the whole experience takes place in a simulated environment on which the designers have complete artistic control.

In SR, designers will not be aware of the environment in which an SR experience will take place. Therefore its design process will need to convey the logic and progression of the experience in an abstract form that does not rely on absolute spatial information (i.e. a task might require users to find an object located on a shelf in the north-western corner of the room) but presents the information in a way that can be dynamically adapted by the system (i.e. users need to find an object located on a planar surface, near a window). In this regard, the design of SR experiences might build on the the insights and methodologies of Procedural Content Generation [9].

Finding a method to obtain an objective measure of the mismatch between the physical environment and the portrayed Virtual Environment represents another challenge. This measure could be useful to compare the same SR experience across different physical environments (performing a similar study to the original work of Usoh et al. [?]). Studying its relationship with the sense of presence would provide more insights into which factors have the most impact on the believability of the experience. For example, is the mis- 
match between physical and virtual objects equally important as the mismatch between the physical location and the virtual counterpart (e.g., a real living room substituted by a different room of another building as opposed to an outdoor location) or not? Is there a limit to the level of mismatch admissible over which an SR experience is no longer feasible in a given physical environment? Finding the answers to these questions will shed further insights on the design space of SR.

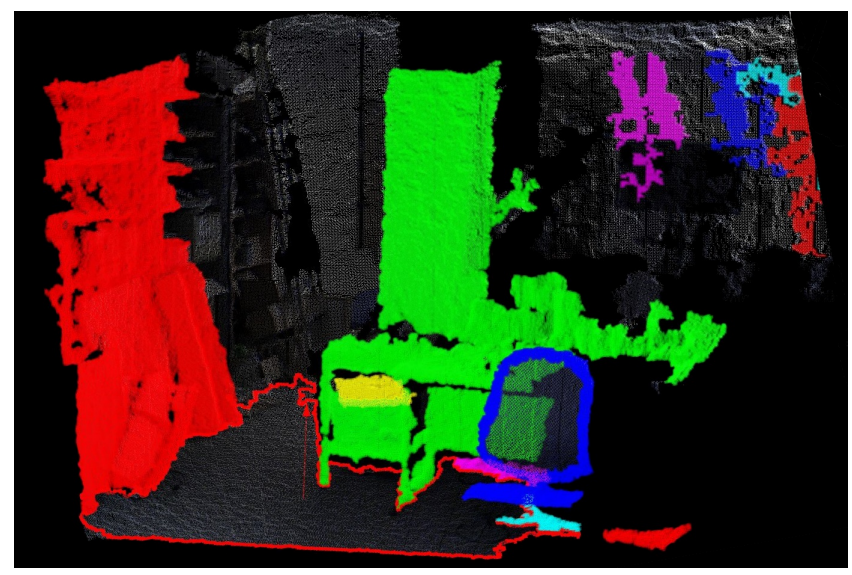

Figure 2: In the picture, an example output of the segmentation of planar surfaces and large object clusters is shown.

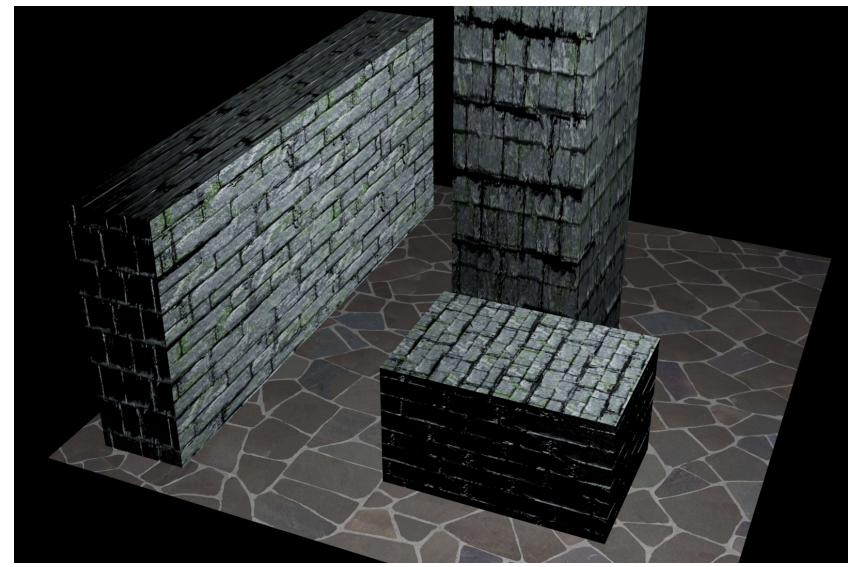

Figure 3: A concept representation of using the output of the previous step in the substitution process (see Fig. 5) to create a 3D environment based on the point cloud captured by a depth camera.

\section{Conclusion}

In this paper we have discussed two main research direction for future explorations of Substitutional Reality. The first, we propose aspects that are yet unexplored, such as a deeper characterisation of the model defined in our previous work; the challenges of locomotion in Substitutional Reality; the combination of immaterial and substituted objects; the possibility of substituting people in multiuser systems. The second direction explores the technical aspect of Substitutional Reality, from systems enabling dynamic adaptation of the Virtual Environment to the layout of the physical environ- ment given as input to the issues of designing and comparing SR experiences.

\section{REFERENCES}

[1] A. Bränzel, C. Holz, D. Hoffmann, D. Schmidt, M. Knaust, P. Lühne, R. Meusel, S. Richter, and P. Baudisch. Gravityspace: Tracking users and their poses in a smart room using a pressure-sensing floor. In Proceedings of the SIGCHI Conference on Human Factors in Computing Systems, CHI '13, pages 725-734, New York, NY, USA, 2013. ACM.

[2] T. Carter, S. A. Seah, B. Long, B. Drinkwater, and S. Subramanian. Ultrahaptics: multi-point mid-air haptic feedback for touch surfaces. In Proceedings of the 26th annual ACM symposium on User interface software and technology, pages 505-514. ACM, 2013.

[3] M. Dou and H. Fuchs. Temporally enhanced 3d capture of room-sized dynamic scenes with commodity depth cameras. In Virtual Reality (VR), 2014 iEEE, pages 39-44. IEEE, 2014.

[4] J. J. Gibson. The theory of affordances. Perceiving, Acting and Knowing, 1977.

[5] H. Hoffmann. Physically touching virtual objects using tactile augmentation enhances the realism of virtual environments. In Virtual Reality Annual International Symposium, VR '08, pages 59-63. IEEE, 1998.

[6] S. Izadi, D. Kim, O. Hilliges, D. Molyneaux, R. Newcombe, P. Kohli, J. Shotton, S. Hodges, D. Freeman, A. Davison, et al. Kinectfusion: real-time $3 \mathrm{~d}$ reconstruction and interaction using a moving depth camera. In Proceedings of the 24th annual ACM Symposium on User Interface Software and Technology, UIST '11, pages 559-568. ACM, 2011.

[7] B. Jones, R. Sodhi, M. Murdock, R. Mehra, H. Benko, A. Wilson, E. Ofek, B. MacIntyre, N. Raghuvanshi, and L. Shapira. Roomalive: Magical experiences enabled by scalable, adaptive projector-camera units. In Proceedings of the 27th Annual ACM Symposium on User Interface Software and Technology, UIST '14, pages 637-644, New York, NY, USA, 2014. ACM.

[8] B. R. Jones, H. Benko, E. Ofek, and A. D. Wilson. Illumiroom: Peripheral projected illusions for interactive experiences. In Proceedings of the SIGCHI Conference on Human Factors in Computing Systems, CHI '13, pages 869-878, New York, NY, USA, 2013. ACM.

[9] R. Khaled, M. J. Nelson, and P. Barr. Design metaphors for procedural content generation in games. In Proceedings of the SIGCHI Conference on Human Factors in Computing Systems, CHI '13, pages 1509-1518, New York, NY, USA, 2013. ACM.

[10] A. Kulik, A. Kunert, S. Beck, R. Reichel, R. Blach, A. Zink, and B. Froehlich. C1x6: a stereoscopic six-user display for co-located collaboration in shared virtual environments. ACM Transactions on Graphics (TOG), 30(6): 188, 2011

[11] R. B. Rusu and S. Cousins. 3d is here: Point cloud library (pcl). In Robotics and Automation (ICRA), 2011 IEEE International Conference on, pages 1-4. IEEE, 2011.

[12] A. Simeone, E. Velloso, and H. Gellersen. Substitutional reality: Using the physical environment to design virtual reality experiences. In Proceedings of the 33rd annual ACM conference on Human factors in computing systems, $\mathrm{CHI}$ ' 15 . ACM, 2015.

[13] Sixense. Stem, 2015 (accessed February 1, 2015). http:// sixense.com/wireless.

[14] R. Sodhi, I. Poupyrev, M. Glisson, and A. Israr. Aireal: Interactive tactile experiences in free air. ACM Trans. Graph., 32(4):134:1-134:10, July 2013

[15] K. Suzuki, S. Wakisaka, and N. Fujii. Substitutional reality system: a novel experimental platform for experiencing alternative reality. Scientific reports, 2, 2012.

[16] M. Usoh, K. Arthur, M. C. Whitton, R. Bastos, A. Steed, M. Slater, and F. P. Brooks Jr. Walking; walking-in-place $i$ flying, in virtual environments. In Proceedings of the 26th Annual Conference on Computer Graphics and Interactive Techniques, pages 359-364. ACM Press/Addison-Wesley Publishing Co., 1999.

[17] M. Usoh, E. Catena, S. Arman, and M. Slater. Using presence questionnaires in reality. Presence, 9(5):497-503, 2000.

[18] YEI Technology. Priovr, 2014 (accessed February 1, 2015). http: //priovr.com. 